\title{
Proposing and Dynamical Analysis of a Hyperjerk Piecewise Linear Chaotic System with Offset Boostable Variable and Hidden Attractors
}

\author{
M. D. Vijayakumar, ${ }^{1}$ Sajjad Shaukat Jamal ${ }^{1 D},{ }^{2}$ Ahmed M. Ali Ali, ${ }^{3}$ \\ Karthikeyan Rajagopal ${ }^{D},{ }^{4}$ Sajad Jafari, ${ }^{5,6}$ and Iqtadar Hussain ${ }^{7}$ \\ ${ }^{1}$ Center for Materials Research, Chennai Institute of Technology, Chennai, India \\ ${ }^{2}$ Department of Mathematics, College of Science, King Khalid University, Abha, Saudi Arabia \\ ${ }^{3}$ Department of Power Mechanics, Babylon Technical Institute, Al-Furat Al-Awsat Technical University, Babylon 51001, Iraq \\ ${ }^{4}$ Centre for Nonlinear Systems, Chennai Institute of Technology, Chennai, Tamilnadu 600069, India \\ ${ }^{5}$ Health Technology Research Institute, Amirkabir University of Technology, 424 Hafez Ave., Tehran 15875-4413, Iran \\ ${ }^{6}$ Department of Biomedical Engineering, Amirkabir University of Technology, 424 Hafez Ave., Tehran 15875-4413, Iran \\ ${ }^{7}$ Department of Mathematics, Statistics and Physics, Qatar University, Doha 2713, Qatar
}

Correspondence should be addressed to Karthikeyan Rajagopal; rkarthiekeyan@gmail.com

Received 14 May 2021; Revised 10 July 2021; Accepted 2 August 2021; Published 9 August 2021

Academic Editor: Eric Campos

Copyright (c) 2021 M. D. Vijayakumar et al. This is an open access article distributed under the Creative Commons Attribution License, which permits unrestricted use, distribution, and reproduction in any medium, provided the original work is properly cited.

Designing chaotic systems with different properties helps to increase our knowledge about real-world chaotic systems. In this article, a piecewise linear (PWL) term is employed to modify a simple chaotic system and obtain a new chaotic model. The proposed model does not have any equilibrium for different values of the control parameters. Therefore, its attractor is hidden. It is shown that the PWL term causes an offset boostable variable. This feature provides more flexibility and controllability in the designed system. Numerical analyses show that periodic and chaotic attractors coexist in some fixed values of the parameters, indicating multistability. Also, the feasibility of the system is approved by designing field programmable gate arrays (FPGA).

\section{Introduction}

Chaotic systems are characterized by sensitivity to initial conditions, known as the butterfly effect, and unpredictability. Recently, the analyzing, designing, and applications of chaotic systems have been progressed in different fields. The existence of chaotic behaviors in real-world systems $[1,2]$ encourages proposing physical [3], biological $[4,5]$, economic [6], and engineering $[7,8]$ chaotic models. Previous studies suggested that chaotic systems have different numbers and geometric forms of equilibria, e.g., chaotic systems with no [9-11], a line [12], a circle [13], a curve [14], and a surface [15] of equilibria. Chaotic oscillators can be applied in functional subsystems of digital and radio-wave communication systems such as compression, filter, encryption, and modulation [16].

Most classical and well-known attractors, such as Lorenz, Rössler, Chua, and Chen, are excited from unstable equilibria. So, there is at least one unstable equilibrium in these attractors' basins of attraction. Hence, they are called self-excited attractors [17]. However, about ten years ago, Leonov et al. showed that the Chua's circuit has another kind of attractor for which its basin of attraction does not collide with unstable manifolds [18]. Such attractors are called hidden attractors. Strange attractors in systems with no equilibria are placed in this category $[19,20]$. Exploring the basin of attraction [21] and parameter switching [22] algorithms can detect the hidden attractors of the system. 
The existence of different stable attractors divides the phase space into different regions demonstrating as the basin of attractions [23-26]. Sometimes attractors coexist with each other and are characterized by the initial conditions. Systems with these separated attractors (and their corresponding basins of attraction) are called multistable [27-29]. Altering a multistable system to a monostable one [30] or synchronization of some multistable systems to luck on a particular attractor [31] are two different controlling approaches related to multistable systems. As there is no analytical way to investigate the detect coexisting attractors, numerical approaches are used in most researches [32]. Also, varying initial conditions, as a bifurcation parameter, may help in finding coexisting attractors, especially in systems with extreme multistability [28, 33].

Piecewise linear (PWL) systems are in the category of switching systems with different affine or nonlinear functions in diverse regions of the state variables [34]. Chua's system is one of the most popular PWL systems, which shows chaotic behavior [35]. Different PWL systems have been proposed with multiscroll chaotic attractors and different numbers of equilibria [36, 37]. Primarily, polynomial approaches have been used to generate such chaotic attractors [38, 39]. As PWL functions can generate various chaotic attractors [40], they can be used in controlling [41] and dynamics editing [42]. Also, error state feedback controllers are proposed, which synchronize master-slave PWL chaotic system [43, 44].

Controlling the amplitude of the chaotic attractors is divided into partial and total control schemes [45]. Moreover, researches showed that these approaches might help to find the multistability in systems [46]. Boosting a state variable through adding a DC offset to the variable (offset boosting) is a property that exists in some chaotic systems [47]. The offset boostable variables of a system can even change from unipolar signal to bipolar one and vice versa [48]. Also, researches have shown that conditional symmetry could be preserved for the asymmetric systems using the offset boosting variables which provides polarity balance in these systems [49].

The remainder of this paper is organized as follows: the proposed chaotic system is introduced and statistically analyzed in Section 2. In Section 3, the system's dynamical properties are analyzed using the bifurcation diagram and Lyapunov exponents diagram. The offset boosting property is studied in Section 4. Section 5 includes the FPGA realization of this system. Finally, the discussion and conclusion of this study are presented in Section 6.

\section{Hidden Chaotic Hyperjerk System with PWL Term}

To study the effect of a PWL term on the dynamical properties of chaotic systems, a simple $4 \mathrm{D}$ chaotic hyperjerk system is considered as [50]

$$
\begin{aligned}
\dot{x} & =y, \\
\dot{y} & =z, \\
\dot{z}= & w, \\
\dot{w}= & -1.02 w+1.64 x^{2}-1.36 y^{2} \\
& +0.28 x y+2.42 x z+1.45,
\end{aligned}
$$

where $x, y, z$, and $w$ are the state variables of this ordinary differential equation. Analyzing the steady state, $(\dot{x}, \dot{y}, \dot{z}, \dot{w})=(0,0,0,0)$, shows that this system does not have any equilibria, and its chaotic responses are in the group of hidden attractors. In this work, this system is modified with one PWL term as

$$
\begin{aligned}
\dot{x}= & y, \\
\dot{y}= & z, \\
\dot{z}= & w, \\
\dot{w}= & -1.02 w+1.64 x^{2}-1.36 y^{2} \\
& +0.28 x y+2.42 x z+1.45+a|y-x-b| .
\end{aligned}
$$

The system has two control parameters ( $a$ and $b$ ) and five nonlinearities (one of them is an absolute function of $x$ and $y, g(x, y)=a|y-x-b|)$. For $a>0$, this system does not have any equilibria. Therefore, its chaotic attractor is hidden. Equation (3) determines the volume contraction rate of system (2):

$$
\nabla V=\frac{\partial \dot{x}}{\partial x}+\frac{\partial \dot{y}}{\partial y}+\frac{\partial \dot{z}}{\partial z}+\frac{\partial \dot{w}}{\partial w}=-1.02
$$

As $\nabla V<0$, the proposed model is dissipative and has the condition of exhibiting bounded attractors. The state spaces of system (2), Figure 1, show that it has chaotic attractor when $a=3.5, b=1$, and $\left(x_{0}, y_{0}, z_{0}, w_{0}\right)=[-2.77,-0.53$, $2.7,-0.34]$. Also, both the piecewise linear functions $g(x, y)$ and the attractor are plotted in the $3 \mathrm{D}$ phase space of the system shown in Figure 2.

It should be noted that the fourth-order Runge-Kutta (RK4) [51] method with time-step $h=0.001$ was used to do the numerical simulations.

\section{Dynamical Analysis}

To analyze the effect of parameters of PWL term in the system dynamics, the bifurcation diagram of system (2) is plotted as the bifurcation parameter changes from -1 to 4.5 in Figure 3(a). The parameter $b$ is 1 , and the initial conditions are $(-2.77,0.53,2.7,-0.34)$. The bifurcation diagram shows that the system exhibits both period-doubling and periodhalving routes to chaos in different parameter values. Also, Lyapunov exponents (LEs) of the system are computed for the same conditions and a runtime of $t=40000 \mathrm{~s}$ in Figure 3(b). To calculate the LEs of the system, the Wolf method [52] is used. The largest Lyapunov exponent (LLE, shown in green) is positive when the bifurcation diagram displays chaotic behavior. 


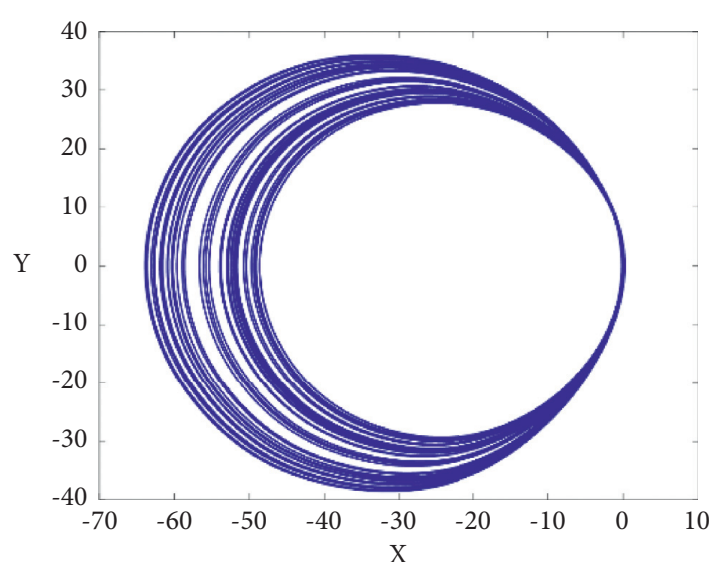

(a)

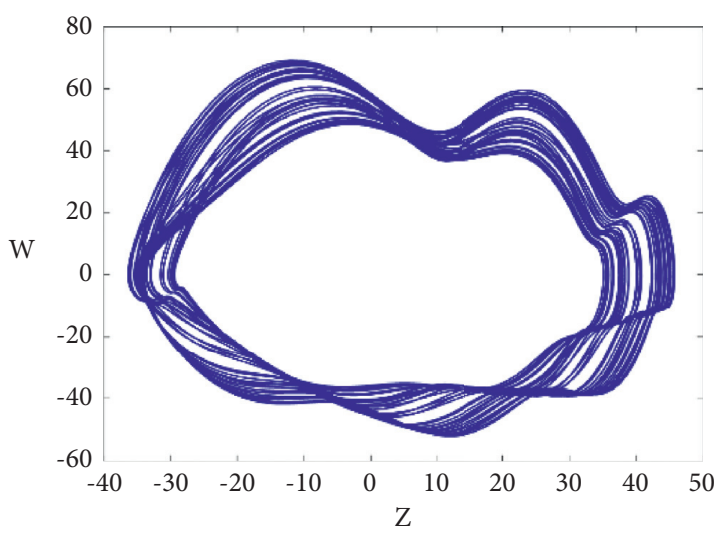

(c)

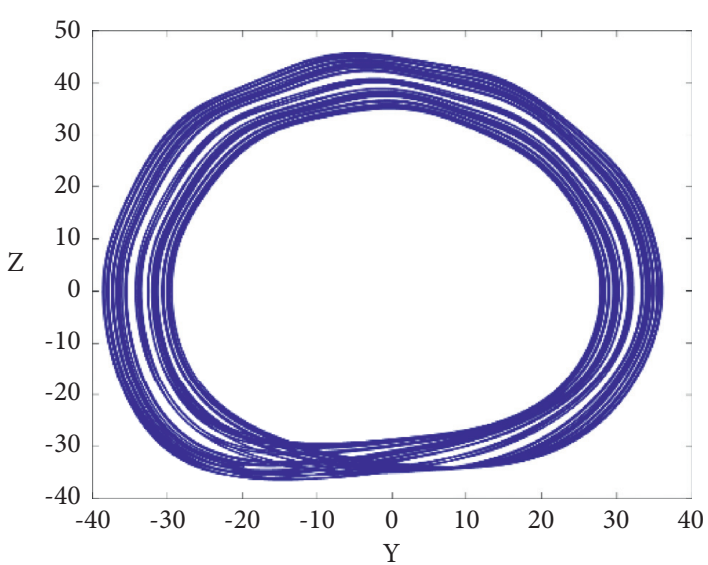

(b)

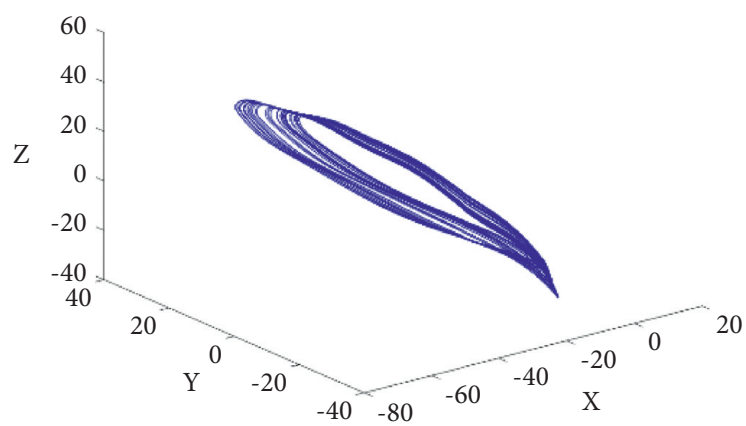

(d)

FIGURE 1: Attractor of system (2) and its projections in (a) $x y$, (b) $y z$, and (c) $z w$ planes and (d) $x y z$ space with $a=3.5$ and $b=1$ and the initial conditions are $\left(x_{0}, y_{0}, z_{0}, w_{0}\right)=(-2.77,0.53,2.7,-0.34)$.

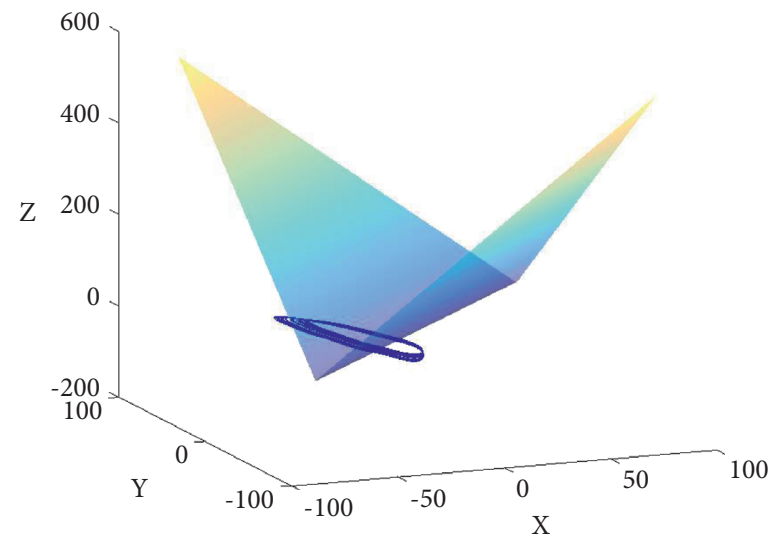

Figure 2: The piecewise linear function of the proposed system when $a=3.5$ and $b=1$ in $x y z$ view. Also, the attractor of the system is plotted in dark blue.

Also, changing the control parameter $b$ results in different dynamical behaviors in this system. To investigate, the bifurcation diagram of the system is plotted in Figure 4(a), in which $b$ changes from -0.2 to 2.8 . Figure 4 (a) shows that different values of this parameter result in period-doubling and period-halving routes to chaos. Figure 4(b) shows the corresponding LEs. Both panels of Figure 4 are plotted when $a=3.5$ and $\left(x_{0}, y_{0}, z_{0}, w_{0}\right)=(-2.77,0.53,2.7,-0.34)$.

Different initial conditions are checked for constant values of the parameters. This system has two coexisting attractors, a chaotic one and a limit cycle one, when $a=3.9$ and $b=1$. Figure 5 displays these attractors in red and blue 


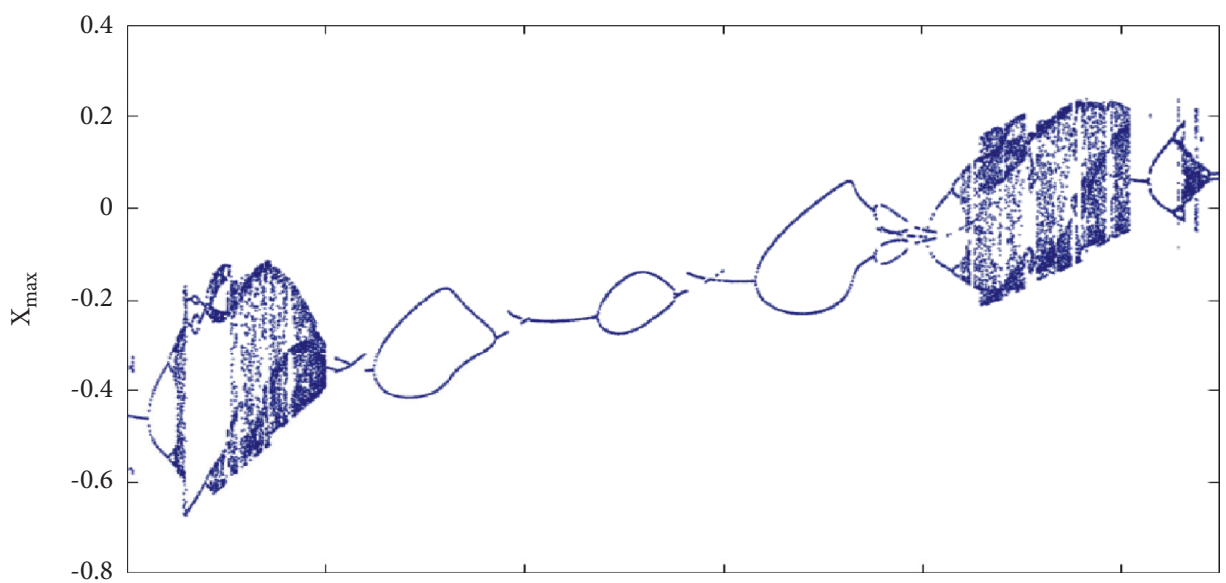

(a)

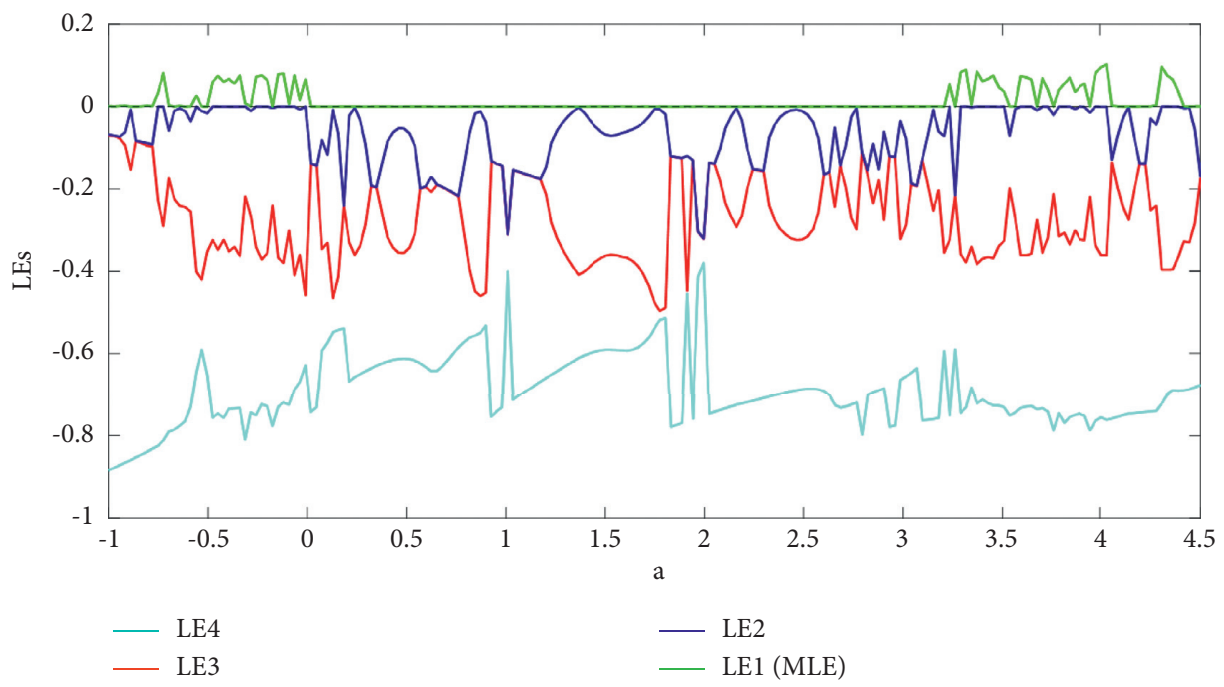

(b)

Figure 3: (a) Bifurcation diagram and (b) Lyapunov exponents of system (2) when parameter $a$ changes and $b=1$. The initial conditions are $(-2.77,0.53,2.7,-0.34)$.

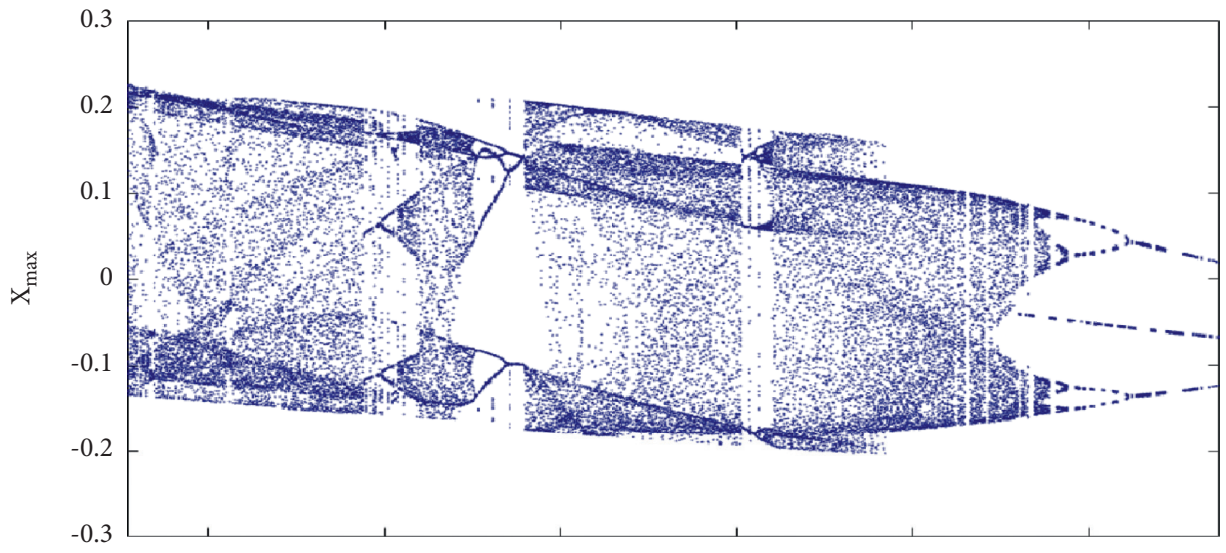

(a)

FIgURE 4: Continued. 


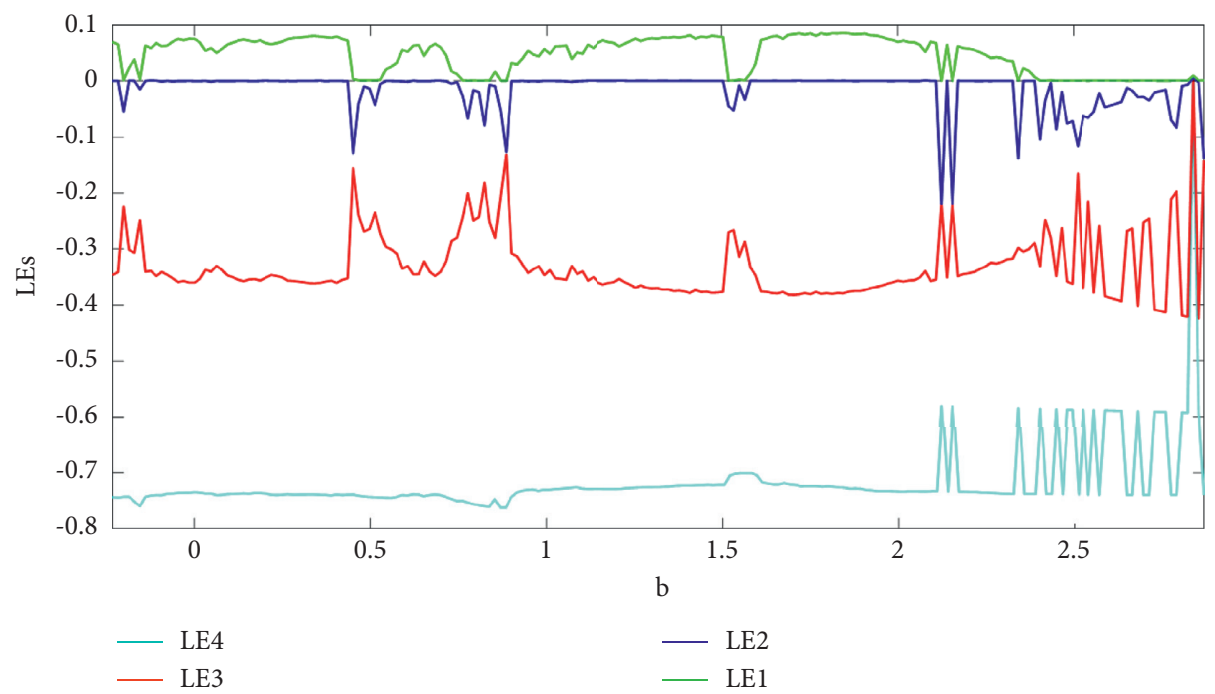

(b)

Figure 4: (a) Bifurcation diagram and (b) Lyapunov exponents of system (2) when parameter $b$ changes and $a=3.5$. The initial conditions are $(-2.77,0.53,2.7,-0.34)$.

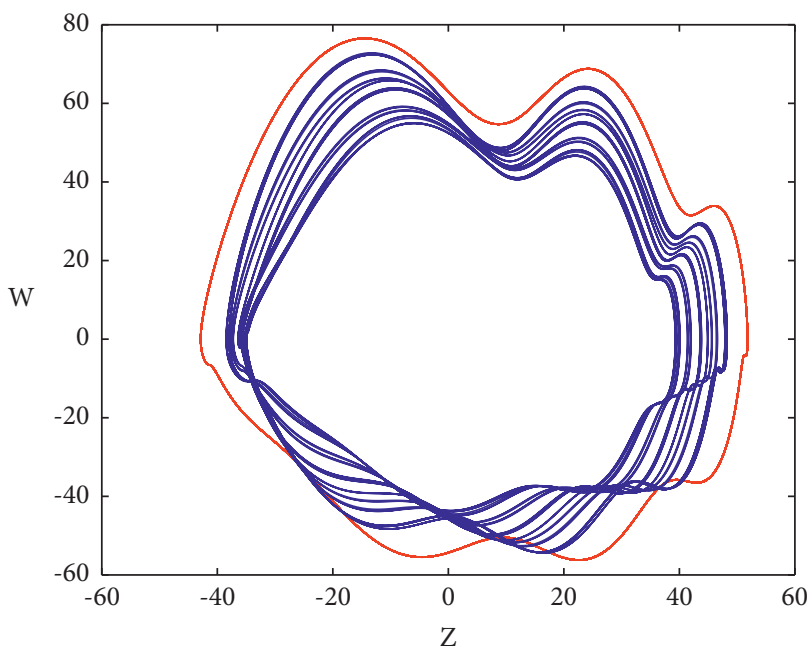

FIgURE 5: The system is multistable when $a=3.9$ and $b=1$. Initial conditions for red and blue colors are $(-2.77,-0.4021,6.2522,-0.0634)$ $(-2.77,-0.4140,-1.5095,-0.3374)$, respectively.

colors for the initial conditions equal to $(-2.77,-0.4021,6.2522,-0.0634)$ and $(-2.77,-0.4140$, $-1.5095,-0.3374)$, respectively.

\section{Offset Boosting of the Attractors}

One or more state variables are offset boostable in some chaotic systems if adding a DC offset to this variable can boost the attractor in its dimension line. In this system, variable $z$ is offset boostable, and by the transformation $z \longrightarrow z+k$ where $k$ is a constant, the system's attractor moves forward and backward in the $z$ dimension. So, equation (2) changes to

$$
\begin{aligned}
\dot{x}= & y, \\
\dot{y}= & z+k, \\
\dot{z}= & w, \\
\dot{w}= & -1.02 w+1.64 x^{2}+\left(-1.36 y^{2}\right) \\
& +0.28 x y+2.42 x(z+k)+1.45+a|y-x-b| .
\end{aligned}
$$

Considering this transformation in system (1), without the PWL term, shows that variable $z$ is not offset boostable in the original system. In this case (Figure 6(a)), the attractor of the system changes from limit cycle to chaotic one as $k$ varies from -3 to 3 , with the step size equal to 0.5 . However, in system (2), changing the parameter $k$ from -30 to 30 boosts the attractor, as shown in Figure 6(b). It should be noted that increasing the parameter $k$ alters the state variable $z$ from a bipolar signal to a unipolar one.

To better represent the effect of the offset boosting parameter, the average of the state variables should be analyzed. The average of offset boostable variables should increase linearly as the parameter $k$ increases, while other state variables' averages should remain constant. Figure 7 (b) shows that these conditions are satisfied in system (2) while the original system does not have this feature. In system (1), $X_{\text {mean }}$ does not remain constant, and $Z_{\text {mean }}$ has some fluctuations.

\section{FPGA Implementation of 4D PWL Hyperjerk System}

Digital implementations of a chaotic system have incredible importance in capturing the main characteristics of nonlinear systems. Recent literature has shown an increasing interest in hardware implementation of nonlinear models 


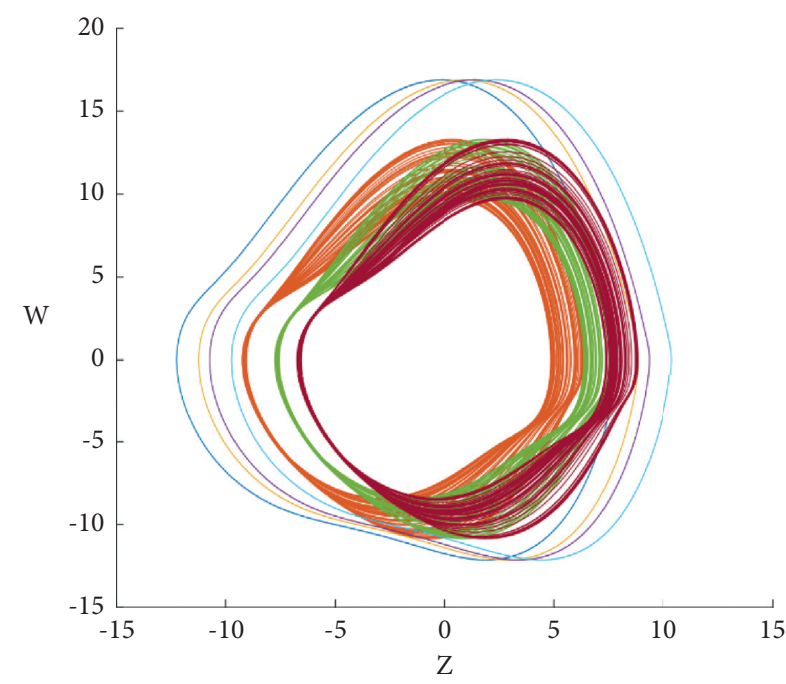

(a)

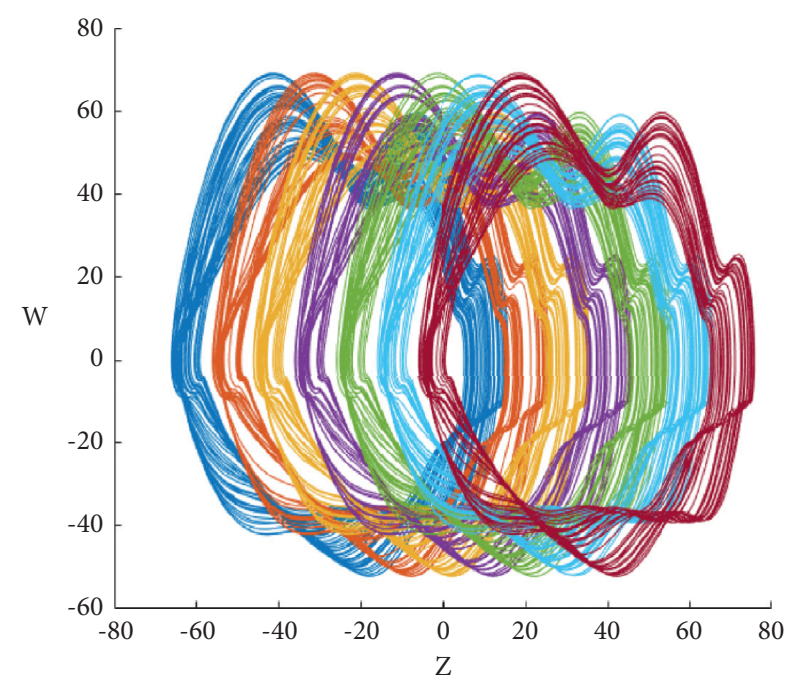

(b)

Figure 6: Projection of systems (1) and (2) in the $z w$ plane. Varying the control parameter $k$, (a) from -3 to 3 in system (1), shows that this system is not offset boostable. However, in (b), when $k$ changes from -30 to 30 in system (2), the system shows offset boostable property. In both panels, $a=3.5, b=1$, and $\left(x_{0}, y_{0}, z_{0}, w_{0}\right)=(-2.77-0.532 .7-0.34)$.

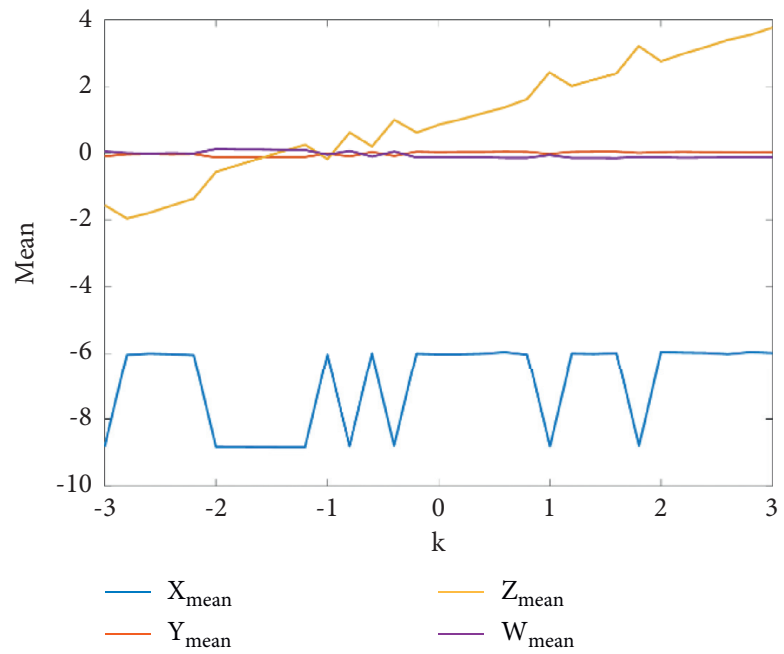

(a)

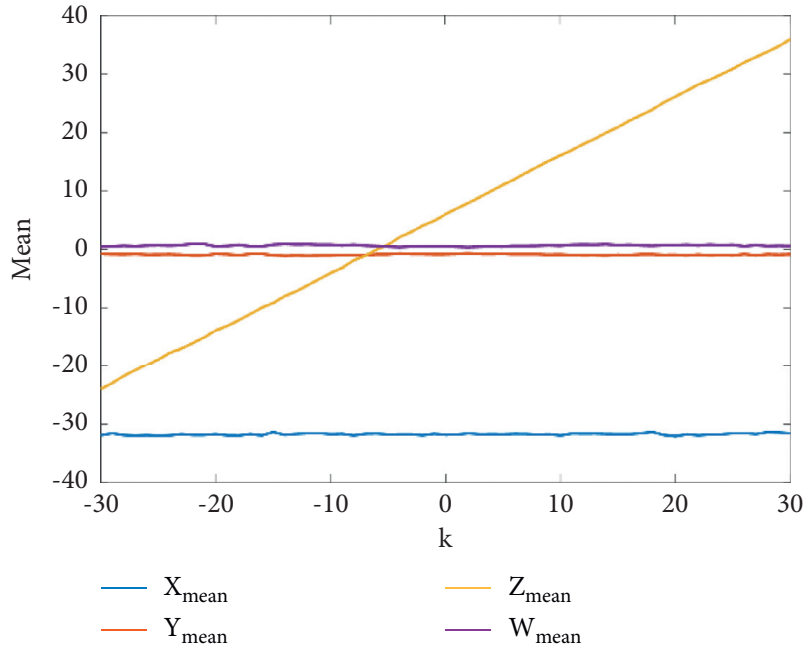

(b)

FiguRE 7: (a) and (b) the state variables' average in systems (1) and (2). (a) The average of variable $z$ does not precisely increase linearly when the control parameter $k$ increases. Also, $X_{\text {mean }}$ does not remain constant. However, in system (2), the linear increase of $Z_{\text {mean }}$ is seen.

[53-57]. Field programmable gate arrays (FPGA) became a vital tool in characterizing the applications of different implementations of chaotic systems. Many references have shown the practical implementation of nonlinear systems using FPGA [58-62]. The main attraction of FPGA is its lower power consumption compared to other hardware platforms [63-67].

The proposed 4D PWL hyperjerk system is implemented in the FPGA platform, which has high throughput and utilizes fewer resources. The system's schematic and power analysis chart show the number of resources used for the implementation and its utilization percentage of power. The phase space diagram of the system is obtained using Xilinx System Generator tool $[64,66,67]$. This tool is integrated with MATLAB software, and a Simulink diagram is designed using Xilinx blocks which are readily available in the system generator tool kit. Xilinx block sets used in the Simulink design are configured according to the IEEE 754 standard with 32/16 (input/output) bits floating-point and latency equal zero. A forward Euler (FE) numerical method is used to design an integrator of each state equation of system (2). The general form of the FE numerical method and its discretized state equation of system (2) are shown in equations (5) and (6), respectively, as 


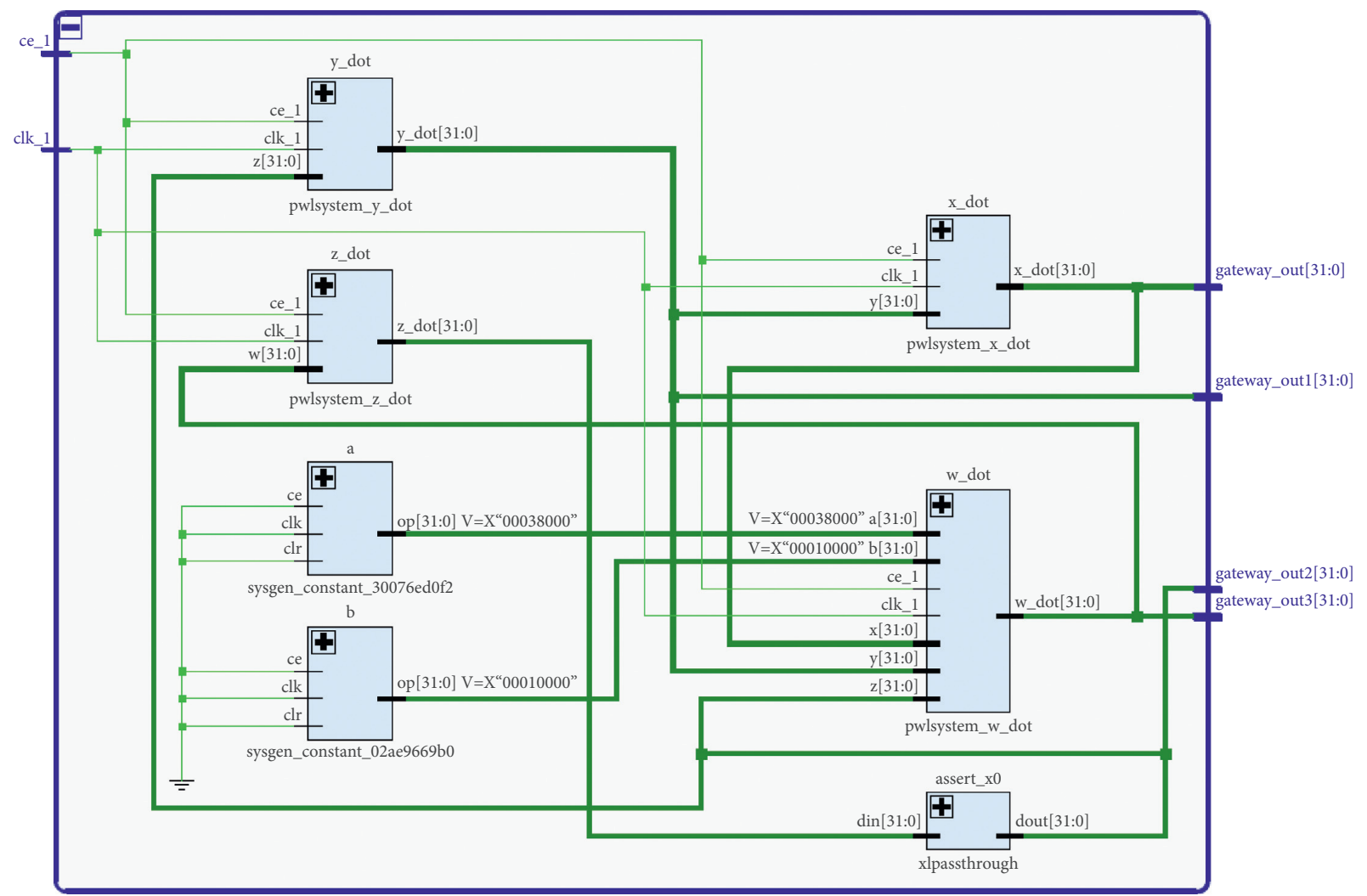

FIGURE 8: Register transfer level schematic of 4D PWL hyperjerk system implemented in Kintex 7 KC705.

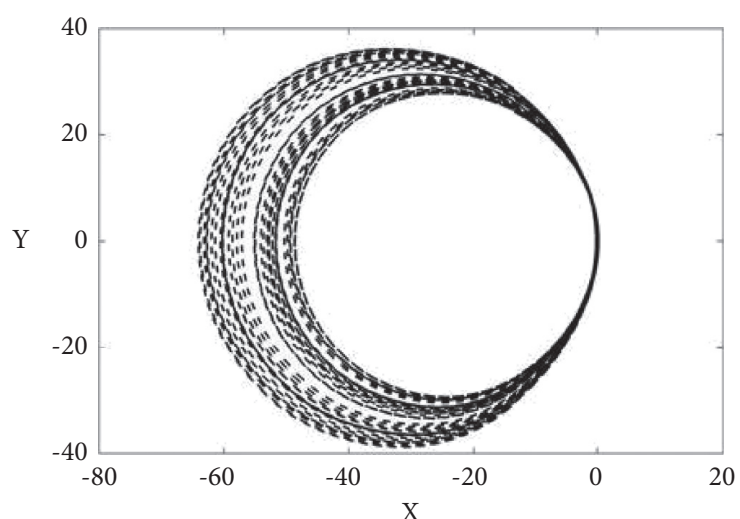

(a)

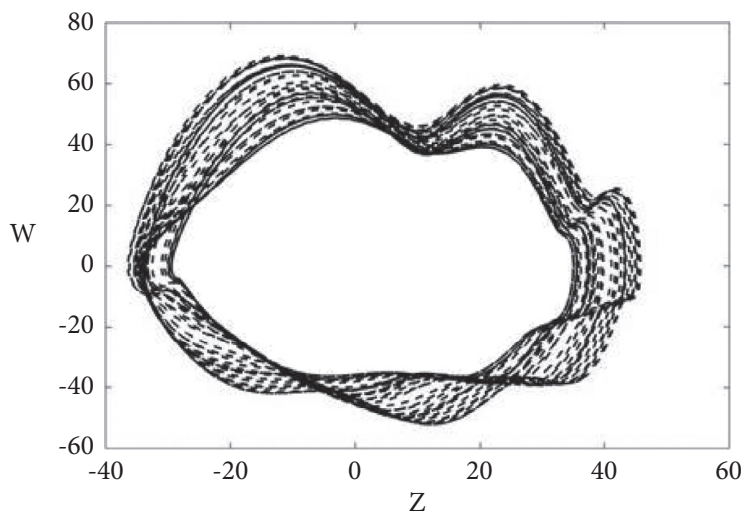

(c)

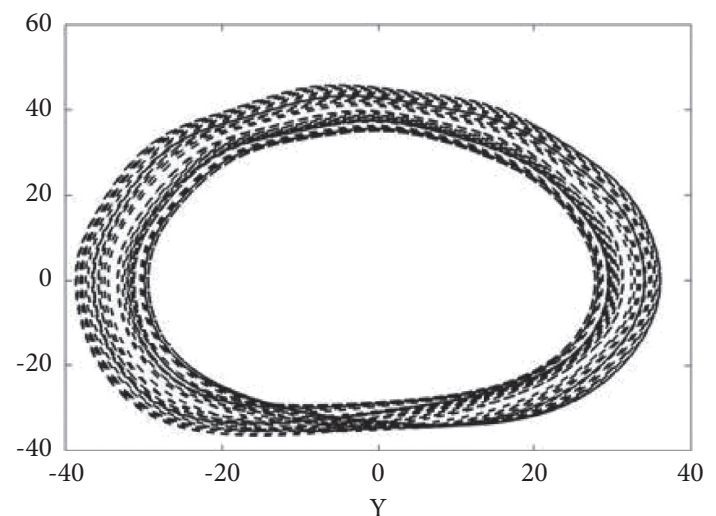

(b)

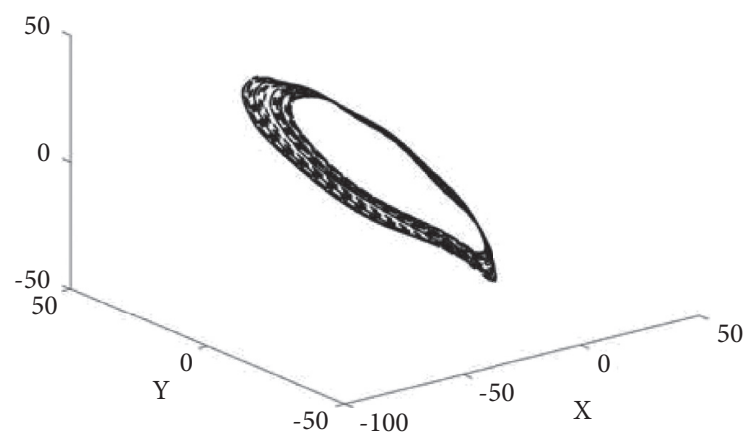

(d)

Figure 9: State space in (a) $x y$, (b) $y z$, (c) $z w$, and (d) $x y z$ of system (2) when $a=3.5$ and $b=1$ with initial conditions $\left(x_{0}, y_{0}, z_{0}, w_{0}\right)=(-2.77,-0.53,2.7,-0.34)$. 
TABLE 1: Resource utilization table for the 4D PWL hyperjerk system implemented in FPGA.

\begin{tabular}{lcccc}
\hline S. no. & Name of resources & Utilization of resources & Total available resources & Percentage of utilization \\
\hline 1 & FF & 256 & 202800 & 0.13 \\
2 & LUT & 753 & 101400 & 0.74 \\
3 & I/O & 129 & 285 & 45.26 \\
5 & DSP48 & 40 & 600 & 6.67 \\
6 & BUFG & 1 & 32 & 3.13 \\
\hline
\end{tabular}

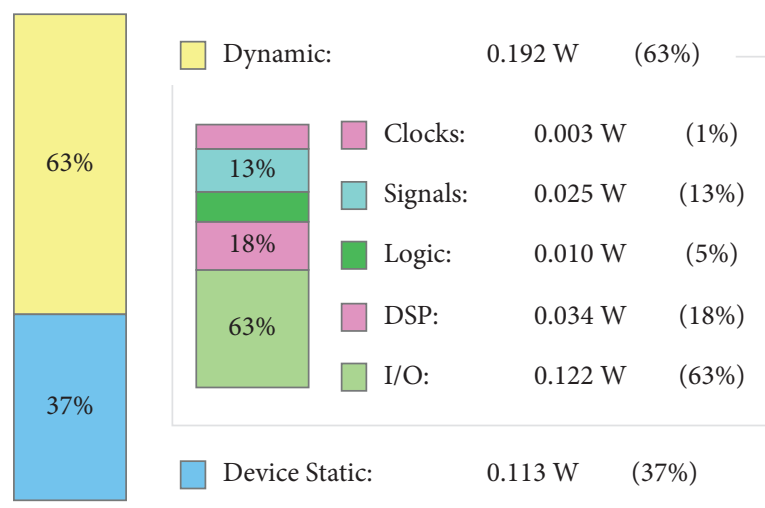

FIgure 10: Power utilization of the FPGA implemented 4D PWL hyperjerk system.

$$
\begin{aligned}
u_{n+1}= & u_{n}+h f\left(u_{n-1}\right), \\
x_{n+1}= & x_{n}+h\left(y_{n-1}\right), \\
y_{n+1}= & y_{n}+h\left(z_{n-1}\right), \\
z_{n+1}= & z_{n}+h\left(w_{n-1}\right), \\
w_{n+1}= & w_{n}+h\left(-1.02 w_{n-1}\right. \\
& +1.64 x_{n-1}^{2}-1.36 y_{n-1}^{2} \\
& +0.28 x_{n-1} y_{n-1} \\
& +2.42 x_{n-1} z_{n-1}+1.45 \\
& \left.+a\left|y_{n-1}-x_{n-1}-b\right|\right) .
\end{aligned}
$$

The step size $h$ is set to 0.001 . Also, the parameter values $a=3.5$ and $b=1$ are generated using Xilinx constant block. The state phase diagram is produced and shown in Figure 8 when $\left(x_{0}, y_{0}, z_{0}, w_{0}\right)=(-2.77,-0.53,2.7,-0.34)$. Following the Simulink design $[56,57]$, the system is then synthesized and implemented through Vivado design tool, and its VHDL or VERILOG description is automatically generated. An elaborated design of system (2) using the Kintex 7 xc7k160t-1fbg484 chip is presented in Figure 9. Hardware-software cosimulation is performed with Kintex $7 \mathrm{KC705}$ kit to prove that the proposed system is implemented using hardware $[66,67]$. The utilization of resources and power is presented in Table 1 and Figure 10, respectively.

\section{Discussion and Conclusion}

About ten years ago, Leonov et al. discovered a kind of attractor in which the basin of attraction did not intersect with neighborhoods of equilibria [68]. Such attractors are different from the previously known ones, which can be excited from unstable equilibria. Investigating hidden attractors in real-world systems has received much interest as they may cause undesired dynamics and stability issues. Systems with no equilibria are an important subgroup of hidden attractors. Therefore, we were motivated to design a system with no equilibria which employed the absolute function as the nonlinear term.

Numerical results showed that taking the polarity information from one term of the proposed system makes it have offset boosting property. Due to this rare feature, the proposed system's attractor is moved through the $z$-axis when the control parameter $k$ is changed as $z \longrightarrow z+k$ in equation (2), while the other state variables' average remained constant. Comparing the proposed system with the original system indicated that this property was inserted through the absolute function. The offset boostable variable $z$ allowed adjusting the location of the attractor in its direction, which provides controllability in this system.

Using the absolute function made the proposed system a piecewise linear (PWL) one. PWL systems mostly consist of two or more affine functions. These affine functions provide the situation in which adding more equilibria and switching surfaces provide multiscroll attractors [34, 37]. Although, in the proposed system, a general case was considered with nonlinear terms that empower the attractors' complexity, this claim can be confirmed by comparing the bifurcation diagrams derived from both these subgroups of PWL systems.

Chaotic systems have been frequently used in encryption block of secure communication systems according to their robustness against noise and attack [69]. Previous studies suggested that the chaos-encrypted images, both grayscale and RGB, have no correlation with the original image [70]. 
Also, the quality of the image does not decrease during the decryption process. Chaotic systems also have been used to generate random numbers in cryptography [71]. To improve the application of chaotic systems, optimization methods can be used [72]. These single and multiobjective optimization methods have used different numerical approaches to increase (or decrease) the Lyapunov exponents [73], Kaplan-Yorke dimension [74], and complexity indexes [75]. Future works can focus on optimizing the indexes of the proposed system to provide its application in image encryption and random number generator.

\section{Data Availability}

All the numerical simulation parameters are mentioned in the respective text part, and there are no additional data requirements for the simulation results.

\section{Conflicts of Interest}

The authors declare no conflicts of interest.

\section{Acknowledgments}

The authors extend their gratitude to the Deanship of Scientific Research at King Khalid University for funding this work through research groups program under grant number R.G.P.1/72/42.

\section{References}

[1] H. D. Abarbanel, R. Brown, J. J. Sidorowich, and L. S. Tsimring, "The analysis of observed chaotic data in physical systems," Reviews of Modern Physics, vol. 65, p. 1331, 1993.

[2] H. Korn and P. Faure, "Is there chaos in the brain? II. experimental evidence and related models," Comptes Rendus Biologies, vol. 326, pp. 787-840, 2003.

[3] R. Rollins and E. Hunt, "Exactly solvable model of a physical system exhibiting universal chaotic behavior," Physical Review Letters, vol. 49, p. 1295, 1982.

[4] K. Aihara, T. Takabe, and M. Toyoda, "Chaotic neural networks," Physics Letters A, vol. 144, pp. 333-340, 1990.

[5] Q. Xu, X. Tan, D. Zhu, H. Bao, Y. Hu, and B. Bao, "Bifurcations to bursting and spiking in the chay neuron and their validation in a digital circuit," Chaos, Solitons \& Fractals, vol. 141, Article ID 110353, 2020.

[6] M. Frank and T. Stengos, "Chaotic dynamics in economic time-series,” Journal of Economic Surveys, vol. 2, pp. 103-133, 1988.

[7] B. Bao, N. Wang, Q. Xu, H. Wu, and Y. Hu, "A simple thirdorder memristive band pass filter chaotic circuit," IEEE Transactions on Circuits and Systems II: Express Briefs, vol. 64, pp. 977-981, 2016.

[8] J. Kengne, G. D. Leutcho, and A. N. K. Telem, "Reversals of period doubling, coexisting multiple attractors, and offset boosting in a novel memristive diode bridge-based hyperjerk circuit," Analog Integrated Circuits and Signal Processing, vol. 101, pp. 379-399, 2019.

[9] S. Jafari, J. Sprott, and S. M. R. H. Golpayegani, "Elementary quadratic chaotic flows with no equilibria," Physics Letters A, vol. 377, pp. 699-702, 2013.
[10] R. D. J. Escalante-González and E. Campos-Cantón, "Generation of chaotic attractors without equilibria via piecewise linear systems," International Journal of Modern Physics C, vol. 28, Article ID 1750008, 2017.

[11] R. D. J. Escalante-González and E. Campos-Cantón, “A class of piecewise linear systems without equilibria with 3-D grid multiscroll chaotic attractors," IEEE Transactions on Circuits and Systems II: Express Briefs, vol. 66, pp. 1456-1460, 2018.

[12] J. P. Singh and B. Roy, "The simplest 4-D chaotic system with line of equilibria, chaotic 2-torus and 3-torus behaviour," Nonlinear Dynamics, vol. 89, pp. 1845-1862, 2017.

[13] T. Gotthans, J. C. Sprott, and J. Petrzela, "Simple chaotic flow with circle and square equilibrium," International Journal of Bifurcation and Chaos, vol. 26, Article ID 1650137, 2016.

[14] K. Barati, S. Jafari, J. C. Sprott, and V. T. Pham, "Simple chaotic flows with a curve of equilibria," International Journal of Bifurcation and Chaos, vol. 26, Article ID 1630034, 2016.

[15] S. Jafari, J. Sprott, V. T. Pham, C. Volos, and C. Li, "Simple chaotic 3D flows with surfaces of equilibria," Nonlinear Dynamics, vol. 86, pp. 1349-1358, 2016.

[16] L. Kocarev, "Chaos-based cryptography: a brief overview," IEEE Circuits and Systems Magazine, vol. 1, pp. 6-21, 2001.

[17] D. Dudkowski, S. Jafari, T. Kapitaniak, N. V. Kuznetsov, G. A. Leonov, and A. Prasad, "Hidden attractors in dynamical systems," Physics Reports, vol. 637, pp. 1-50, 2016.

[18] M. F. Danca, N. Kuznetsov, and G. Chen, "Unusual dynamics and hidden attractors of the rabinovich-fabrikant system," Nonlinear Dynamics, vol. 88, pp. 791-805, 2017.

[19] P. Li, T. Zheng, C. Li, X. Wang, and W. Hu, "A unique jerk system with hidden chaotic oscillation," Nonlinear Dynamics, vol. 86, pp. 197-203, 2016.

[20] C. Li and J. C. Sprott, "Coexisting hidden attractors in a 4-D simplified lorenz system," International Journal of Bifurcation and Chaos, vol. 24, Article ID 1450034, 2014.

[21] M. F. Danca, P. Bourke, and N. Kuznetsov, "Graphical structure of attraction basins of hidden chaotic attractors: the rabinovich-fabrikant system," International Journal of Bifurcation and Chaos, vol. 29, Article ID 1930001, 2019.

[22] M. F. Danca, N. V. Kuznetsov, and G. Chen, "Approximating hidden chaotic attractors via parameter switching," Chaos: An Interdisciplinary Journal of Nonlinear Science, vol. 28, Article ID 013127, 2018.

[23] X. Wang and G. Chen, "A chaotic system with only one stable equilibrium," Communications in Nonlinear Science and Numerical Simulation, vol. 17, pp. 1264-1272, 2012.

[24] Q. Li, H. Zeng, and J. Li, "Hyperchaos in a 4D memristive circuit with infinitely many stable equilibria," Nonlinear Dynamics, vol. 79, pp. 2295-2308, 2015.

[25] Q. Xu, Y. Lin, B. Bao, and M. Chen, "Multiple attractors in a non-ideal active voltage-controlled memristor based chua's circuit," Chaos, Solitons \& Fractals, vol. 83, pp. 186-200, 2016.

[26] T. Kapitaniak and G. A. Leonov, Multistability: Uncovering Hidden Attractors, Springer, Berlin, Germany, 2015.

[27] P. Sharma, M. Shrimali, A. Prasad, N. Kuznetsov, and G. Leonov, "Control of multistability in hidden attractors," The European Physical Journal-Special Topics, vol. 224, pp. 1485-1491, 2015.

[28] M. Chen, Y. Feng, H. Bao, B. Bao, H. Wu, and Q. Xu, "Hybrid state variable incremental integral for reconstructing extreme multistability in memristive jerk system with cubic nonlinearity," Complexity, vol. 2019, Article ID 8549472, 16 pages, 2019.

[29] B. Bao, Y. Zhu, C. Li, H. Bao, and Q. Xu, "Global multistability and analog circuit implementation of an adapting synapse- 
based neuron model," Nonlinear Dynamics, vol. 101, pp. 1105-1118, 2020.

[30] R. Sevilla-Escoboza, G. Huerta-Cuéllar, R. Jaimes-Reátegui et al., "Error-feedback control of multistability," Journal of the Franklin Institute, vol. 354, pp. 7346-7358, 2017.

[31] R. Sevilla-Escoboza, J. Buldú, A. Pisarchik, S. Boccaletti, and R. Gutiérrez, "Synchronization of intermittent behavior in ensembles of multistable dynamical systems," Physical Review E, vol. 91, Article ID 32902, 2015.

[32] N. Kuznetsov and G. Leonov, "Hidden attractors in dynamical systems: systems with no equilibria, multistability and coexisting attractors," IFAC Proceedings Volumes, vol. 47, pp. 5445-5454, 2014.

[33] A. Pisarchik, R. Jaimes-Reátegui, C. Rodríguez-Flores, J. García-López, G. Huerta-Cuellar, and F. Martín-Pasquín, "Secure chaotic communication based on extreme multistability," Journal of the Franklin Institute, vol. 358, pp. 2561-2575, 2021.

[34] R. D. J. Escalante-González, E. Campos-Cantón, and M. Nicol, "Generation of multi-scroll attractors without equilibria via piecewise linear systems," Chaos: An Interdisciplinary Journal of Nonlinear Science, vol. 27, Article ID 53109, 2017.

[35] E. Campos, "Derivation of a continuous time dynamic planar system with two unstable foci from a three-dimensional chaotic piecewise linear system," Chaos: An Interdisciplinary Journal of Nonlinear Science, vol. 30, Article ID 53114, 2020.

[36] C. Li, J. C. Sprott, W. Thio, and H. Zhu, "A new piecewise linear hyperchaotic circuit," IEEE Transactions on Circuits and Systems II: Express Briefs, vol. 61, pp. 977-981, 2014.

[37] F. Delgado-Aranda, I. Campos-Cantón, E. Tristán-Hernández, and P. Salas-Castro, "Hidden attractors from the switching linear systems," Revista Mexicana de Física, vol. 66, pp. 683-691, 2020.

[38] J. López-Rentería, E. Campos-Cantón, B. Aguirre-Hernández, and G. Fernández-Anaya, "A monoparametric family of piecewise linear systems to generate scroll attractors via pathconnected set of polynomials," International Journal of Bifurcation and Chaos, vol. 31, Article ID 2150034, 2021.

[39] B. Aguirre-Hernández, E. Campos-Cantón, J. A. LópezRenteria, and E. D. González, "A polynomial approach for generating a monoparametric family of chaotic attractors via switched linear systems," Chaos, Solitons \& Fractals, vol. 71, pp. 100-106, 2015.

[40] Z. Njitacke, T. Fozin, L. K. Kengne, G. Leutcho, E. M. Kengne, and J. Kengne, "Multistability and its annihilation in the chua's oscillator with piecewise-linear nonlinearity," Chaos Theory and Applications, vol. 2, pp. 77-89, 2020.

[41] J. Lü, T. Zhou, G. Chen, and X. Yang, "Generating chaos with a switching piecewise-linear controller," Chaos: An Interdisciplinary Journal of Nonlinear Science, vol. 12, pp. 344-349, 2002.

[42] C. Li, T. Lei, X. Wang, and G. Chen, "Dynamics editing based on offset boosting," Chaos: An Interdisciplinary Journal of Nonlinear Science.vol. 30, Article ID 63124, 2020.

[43] J. R. Pulido-Luna, J. A. López-Renteria, and N. R. Cazarez-Castro, "Chaos synchronization by an observer-based active control," 2004.

[44] J. R. Pulido-Luna, J. A. López-Rentería, and N. R. CazarezCastro, "Design of a nonhomogeneous nonlinear synchronizer and its implementation in reconfigurable hardware," Mathematical and Computational Applications, vol. 25, p. 51, 2020.
[45] C. Li, J. C. Sprott, Z. Yuan, and H. Li, "Constructing chaotic systems with total amplitude control," International Journal of Bifurcation and Chaos, vol. 25, Article ID 1530025, 2015.

[46] C. Li, W. Hu, J. C. Sprott, and X. Wang, "Multistability in symmetric chaotic systems," The European Physical Journal Special Topics, vol. 224, pp. 1493-1506, 2015.

[47] C. Li, X. Wang, and G. Chen, "Diagnosing multistability by offset boosting," Nonlinear Dynamics, vol. 90, pp. 1335-1341, 2017.

[48] C. Li and J. C. Sprott, "Variable-boostable chaotic flows," Optik, vol. 127, pp. 10389-10398, 2016.

[49] C. Li, J. C. Sprott, Y. Liu, Z. Gu, and J. Zhang, "Offset boosting for breeding conditional symmetry," International Journal of Bifurcation and Chaos, vol. 28, Article ID 1850163, 2018.

[50] S. Panahi, V. T. Pham, K. Rajagopal, O. Boubaker, and S. Jafari, "A new four-dimensional chaotic system with no equilibrium point," Recent Advances in Chaotic Systems and Synchronization, Elsevier, Amsterdam, The Netherlands, pp. 63-76, 2019.

[51] O. Guillén-Fernández, M. F. Moreno-López, and E. TleloCuautle, "Issues on applying one-and multi-step numerical methods to chaotic oscillators for FPGA implementation," Mathematics, vol. 9, p. 151, 2021.

[52] A. Wolf, J. B. Swift, H. L. Swinney, and J. A. Vastano, "Determining lyapunov exponents from a time series," Physica D: Nonlinear Phenomena, vol. 16, pp. 285-317, 1985.

[53] M. F. Tolba, A. H. Elsafty, M. Armanyos, L. A. Said, A. H. Madian, and A. G. Radwan, "Synchronization and fpga realization of fractional-order izhikevich neuron model," Microelectronics Journal, vol. 89, pp. 56-69, 2019.

[54] M. Tuna, M. Alçın, I. Koyuncu, C. B. Fidan, and I. Pehlivan, "High speed FPGA-based chaotic oscillator design," Microprocessors and Microsystems, vol. 66, pp. 72-80, 2019.

[55] M. Tuna and C. B. Fidan, "Electronic circuit design, implementation and FPGA-based realization of a new 3D chaotic system with single equilibrium point," Optik, vol. 127, pp. 11786-11799, 2016.

[56] K. Rajagopal, L. Guessas, A. Karthikeyan, A. Srinivasan, and G. Adam, "Fractional order memristor no equilibrium chaotic system with its adaptive sliding mode synchronization and genetically optimized fractional order PID synchronization," Complexity, vol. 2017, Article ID 1892618, 19 pages, 2017.

[57] K. Rajagopal, F. Nazarimehr, A. Karthikeyan, A. Srinivasan, and S. Jafari, "CAMO: self-excited and hidden chaotic flows," International Journal of Bifurcation and Chaos, vol. 29, Article ID 1950143, 2019.

[58] S. Sadoudi, M. S. Azzaz, M. Djeddou, and M. Benssalah, “An FPGA real-time implementation of the chen's chaotic system for securing chaotic communications," International Journal of Nonlinear Science, vol. 7, pp. 467-474, 2009.

[59] L. Ávalos-Ruiz, C. Zúñiga-Aguilar, J. Gómez-Aguilar, R. Escobar-Jiménez, and H. Romero-Ugalde, "FPGA implementation and control of chaotic systems involving the variable-order fractional operator with mittag-leffler law," Chaos, Solitons \& Fractals, vol. 115, pp. 177-189, 2018.

[60] I. Koyuncu, A. T. Ozcerit, and I. Pehlivan, "Implementation of FPGA-based real time novel chaotic oscillator," Nonlinear Dynamics, vol. 77, pp. 49-59, 2014.

[61] E. Tlelo-Cuautle, V. Carbajal-Gomez, P. Obeso-Rodelo, J. Rangel-Magdaleno, and J. C. Nunez-Perez, "FPGA realization of a chaotic communication system applied to image processing," Nonlinear Dynamics, vol. 82, pp. 1879-1892, 2015. 
[62] H. Chen, S. He, A. D. P. Azucena et al., "A multistable chaotic jerk system with coexisting and hidden attractors: dynamical and complexity analysis, FPGA-based realization, and chaos stabilization using a robust controller," Symmetry, vol. 12, p. 569, 2020.

[63] B. Karakaya, A. Gülten, and M. Frasca, "A true random bit generator based on a memristive chaotic circuit: analysis, design and FPGA implementation," Chaos, Solitons \& Fractals, vol. 119, pp. 143-149, 2019.

[64] A. Karthikeyan and K. Rajagopal, "FPGA implementation of fractional-order discrete memristor chaotic system and its commensurate and incommensurate synchronisations," Pramana, vol. 90, p. 14, 2018.

[65] Q. Ding, J. Pang, J. Fang, and X. Peng, "Designing of chaotic system output sequence circuit based on FPGA and its applications in network encryption card," International Journal of Innovative Computing, Information and Control, vol. 3, pp. 449-456, 2007.

[66] K. Rajagopal, L. Guessas, S. Vaidyanathan, A. Karthikeyan, and A. Srinivasan, "Dynamical analysis and FPGA implementation of a novel hyperchaotic system and its synchronization using adaptive sliding mode control and genetically optimized PID control," Mathematical Problems in Engineering, vol. 2017, Article ID 7307452, 14 pages, 2017.

[67] K. Rajagopal, A. Karthikeyan, and A. K. Srinivasan, "FPGA implementation of novel fractional-order chaotic systems with two equilibriums and no equilibrium and its adaptive sliding mode synchronization," Nonlinear Dynamics, vol. 87, pp. 2281-2304, 2017.

[68] G. Leonov, N. Kuznetsov, and V. Vagaitsev, "Localization of hidden chua's attractors," Physics Letters A, vol. 375, pp. 2230-2233, 2011.

[69] E. García-Guerrero, E. Inzunza-González, O. López-Bonilla, J. Cárdenas-Valdez, and E. Tlelo-Cuautle, "Randomness improvement of chaotic maps for image encryption in a wireless communication scheme using PIC-microcontroller via zigbee channels," Chaos, Solitons \& Fractals, vol. 133, Article ID 109646, 2020.

[70] A. Sambas, S. Vaidyanathan, E. Tlelo-Cuautle et al., "A 3-D multistable system with a peanut-shaped equilibrium curve: circuit design, FPGA realization, and an application to image encryption," IEEE Access, vol. 8, pp. 137116-137132, 2020.

[71] S. Vaidyanathan, A. Sambas, B. Abd-El-Atty et al., "A 5-D multistable hyperchaotic two-disk dynamo system with no equilibrium point: circuit design, FPGA realization and applications to TRNGs and image encryption," IEEE Access, vol. 9, 2021.

[72] E. Tlelo-Cuautle, L. G. De La Fraga, O. Guillén-Fernández, and A. Silva-Juárez, Optimization of Integer/Fractional Order Chaotic Systems by Metaheuristics and Their Electronic Realization, CRC Press, Boca Raton, FL, USA, 2021.

[73] A. Silva-Juárez, C. J. Morales-Pérez, L. G. de la Fraga, E. TleloCuautle, and J. de Jesús Rangel-Magdaleno, "On maximizing the positive lyapunov exponent of chaotic oscillators applying DE and PSO," International Journal of Dynamics and Control, vol. 7, pp. 1157-1172, 2019.

[74] A. Silva-Juárez, E. Tlelo-Cuautle, L. G. de la Fraga, and R. Li, "Optimization of the kaplan-yorke dimension in fractionalorder chaotic oscillators by metaheuristics," Applied Mathematics and Computation, vol. 394, Article ID 125831, 2021.

[75] M. Sadeghpour, H. Salarieh, and A. Alasty, "Minimum entropy control of chaos via online particle swarm optimization method," Applied Mathematical Modelling, vol. 36, pp. 3931-3940, 2012. 